\title{
Hot-Band Anti-Stokes Fluorescence Properties of Alexa Fluor 568
}

\author{
Tamás Gajdos ${ }^{1}$ • Béla Hopp ${ }^{1}$ · Miklós Erdélyi ${ }^{1}$ (]
}

Received: 12 December 2019 / Accepted: 20 January 2020 / Published online: 29 Febuary 2020

(c) The Author(s) 2020

\begin{abstract}
Hot-band absorption and anti-Stokes emission properties of an organic fluorescent dye, Alexa Fluor 568, were characterized and compared with those of Rhodamine 101. The comparison of the properties (e.g., quantum efficiency, spectral distribution, thermal properties, and fluorescence lifetime) between the two dyes confirms that both dyes undergo the same process when excited in the red spectral region. Possible undesirable crosstalk effects and applications in dSTORM microscopy were demonstrated and discussed.
\end{abstract}

Keywords Upconversion · Hot band absorption · Anti-Stokes fluorescence

\section{Introduction}

The emission spectra of fluorescent dyes can be elucidated via radiative/non-radiative transitions between the electronic states and internal conversions. Typically, the process begins with a single photon absorption when the molecule is excited to its higher $S_{1}$ or $S_{2}$ electronic states from the $S_{0}$ ground state. Due to vibrations, the electronic states are broadened; after the photon absorption step, the molecule rapidly relaxes to its lowest vibrational level of $S_{1}$ (Kasha's rule) [1]. Internal conversion is significantly faster $(<1$ ps) than the fluorescence lifetime (1-10 ns) of an excited state. During the fluorescence step, the molecule returns to its ground $S_{0}$ state and emits a single fluorescence photon. Because of the energy loss due to internal conversion, the emitted photon has a longer wavelength, which is called the Stokes shift [2], and the energy difference is typically converted into heat.

However, during the anti-Stokes fluorescence, the emitted photon has higher energy (lower wavelength) than the excitation photon. This process requires some additional energy. The thermodynamic background of this process was highly debated in 1946 by Pringsheim and Vavilov and was eventually solved by Landau in the same year [3]. Several types of anti-Stokes fluorescence pathways can be separated

Miklós Erdélyi

erdelyi@ titan.physx.u-szeged.hu

1 Department of Optics and Quantum Electronics, University of Szeged, Dóm tér 9., Szeged 6720, Hungary based on the excitation model and the source of the missing energy. The latter can come from the absorption of multiple photons or from the heat-populated vibrational states. During two-photon absorption process, a virtual state is assumed to exist between $S_{0}$ and $S_{1}$ to excite the molecule. This is a nonlinear third-order process that was originally predicted in 1931 [4] and experimentally was first demonstrated by laser excitation in 1961 [5]. Because the probability of the two-photon absorption process is low, high energy excitation must be used to ensure the sufficient number of emitted photons.

The triplet-triplet annihilation is a similar process, when the triplet $T_{1}$ level is used as an intermediate energy storage state. During this process, two excited molecules undergo energy transfer. One returns to its ground $S_{0}$ state while the other one excites to the $S_{1}$ state. As a result, the emission spectrum exhibits fluorescence characteristics. This process is also called P-type delayed fluorescence [6, 7].

There are two single photon excitation models that produce anti-Stokes fluorescence. In the first model, molecules that are excited to the $T_{1}$ state are considered. Because of the long lifetime of the triplet state, there is a possibility for the molecule to cross into the $S_{1}$ state via temperature activation (reverse intersystem crossing). During the relaxation, emission can occur from the $S_{1}$ or $T_{1}$ states depending on whether the reverse intersystem crossing occurred. Because it was first observed in eosin [8] and the lifetime of the process concurs with phosphorescence, it is called Type-E delayed fluorescence.

In the second single-photon model, the molecule is excited from a higher vibrational state of $S_{0}$ to $S_{1}$. Because at thermal equilibrium the occupation of the 
vibrational levels follows the Boltzmann distribution, the possibility of absorption from higher energy levels is low and temperature-dependent. However, the lifetime and emission spectra are not affected. The hot-band absorption has been observed with several organic dyes: Rhodamine 101 (Rh101) [9], Rh6G [10], Rh640 [11], RhB [12], Oxazine 1 [13], and cyanide-type [14]. Because of the nature of the initial state, the first applications were based around laser cooling [9, 15-18]. Here, we report the anti-Stokes fluorescence properties of Alexa Fluor 568 (AF568) when it is excited in the red-edge region of its absorption spectrum. Although AF568 is a widely used fluorescence dye in confocal and super-resolution microscopy, to our knowledge, its anti-Stokes emission properties have not been studied.

Assuming a two-channel two-colour super-resolution dSTORM imaging assay with a sample simultaneously labelled with AF568 and AF647 dyes (Fig. 1), the intensity of the captured anti-Stokes images (in the 600/37 channel) when excited with $640 \mathrm{~nm}$ laser is comparable to a weak single molecule fluorescence signal of the AF647 dye in the normal long pass emission range. For overlapping structures, the static anti-Stokes signal can introduce a structured background. Due to the spatial shift of localizations towards the peak of the structured background, the final image can be distorted [19]. To minimize this effect the properties of the AF568 anti-Stokes fluorescence had to be specified. To distinguish between the possible excitation models, the relationships between excitation power, excitation wavelength, and emission intensity were measured.

\section{Results}

\section{Fluorescence Intensity}

First, we determined the function between the excitation power and emitted intensity at different excitation wavelengths $(634,640$, and $647 \mathrm{~nm})$. The output power of each source was calibrated and measured after the microscope objective (Nikon CFI Plan Apo Lambda 60x oil). Both Rh101 and AF568 dyes were prepared in a $10^{-4} \mathrm{M}$ solution of methanol and PBS (1:1). Because of the low emission intensity, an additional bandpass emission filter (Semrock 582/75) was used after the main dichroic mirror (Semrock Di01-R405/488/532/635) and quad-band bandpass emission filter (Semrock FF01-446/510/581/703). For each laser setting, a single frame was captured with an exposure time of $100 \mathrm{~ms}$ using an EMCCD camera (Andor iXon 897). The lasers were fibre coupled, collimated, and expanded before they were focused into the back focal plane of the microscope objective. Assuming a Gaussian beam shape in the image plane, two Gaussian distribution curves were fitted on the captured images along each axis. Using the fitted parameters, the volume under the two-dimensional Gaussian distribution was calculated, and the background noise
Fig. 1 dSTORM measurement of a double-stained drosophila myofibril excited with a $640 \mathrm{~nm}$ laser. A total of 10000 frames were acquired in the Dual Gain mode with an exposure time of $20 \mathrm{~ms}$ using a single Andor Zyla 4.2 sCMOS detector with Cairn OptoSplit II+Bypass. The sample is labelled with b2AF568 and tropomyosin-AF647 [20]. a In the 600/37 emission channel, the anti-Stokes emission of AF568 is clearly visible in the I-bands $(\approx 500$ counts). b In the captured frame from the 647 high-pass emission channel, the blinking events of the AF647 in H-zone $(\approx 1800$ counts) and the crosstalk from the AF568 can be seen in the I-band. c The double lines of tropomyosin in the $\mathrm{H}$-zone can be seen in the reconstructed super-resolved image. d The merged image from (a) and (c)

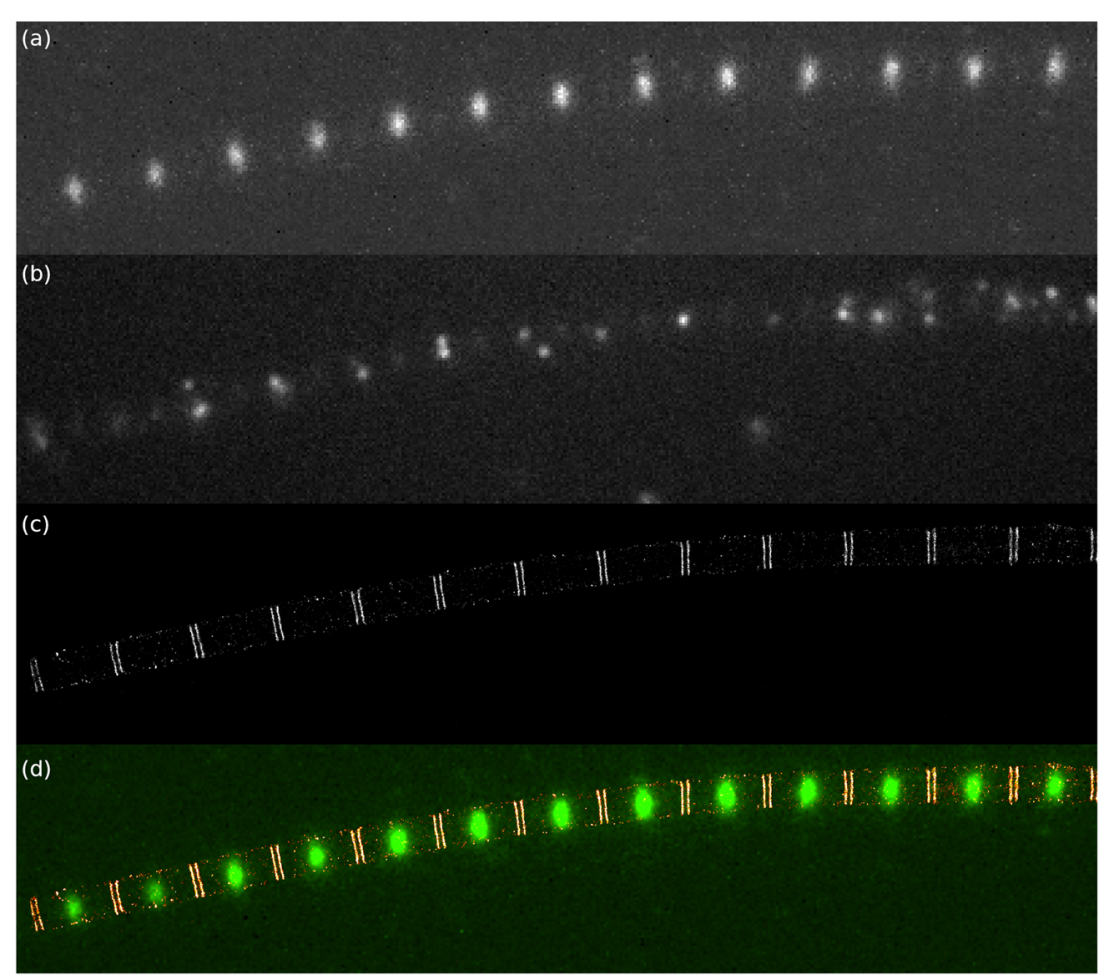


was subtracted. This value was used to indicate the intensity of the anti-Stokes fluorescence.

Expectedly, the absorption cross section is lower for both dyes when excited with longer wavelengths because there are fewer number of fluorophores with high-enough excitable vibrational states in the excited volume (Fig. 2). The measured intensity shows a linear dependence at the lower excitation power range $(<5 \mathrm{~mW})$. The nonlinear feature at higher excitation power is related to the depletion

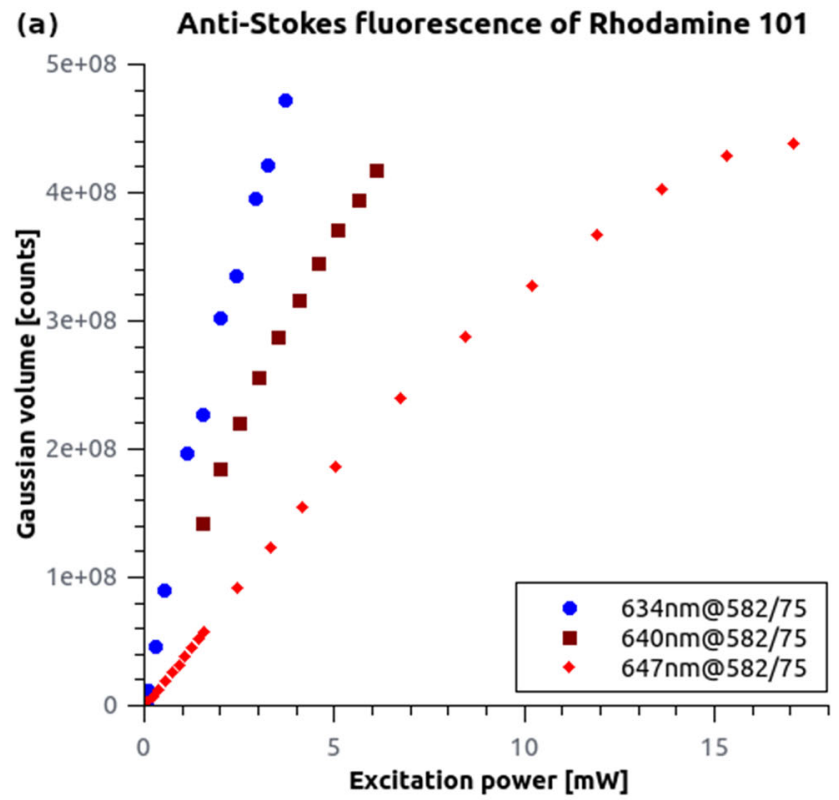

(b) Anti-Stokes fluorescence of Alexa Fluor 568

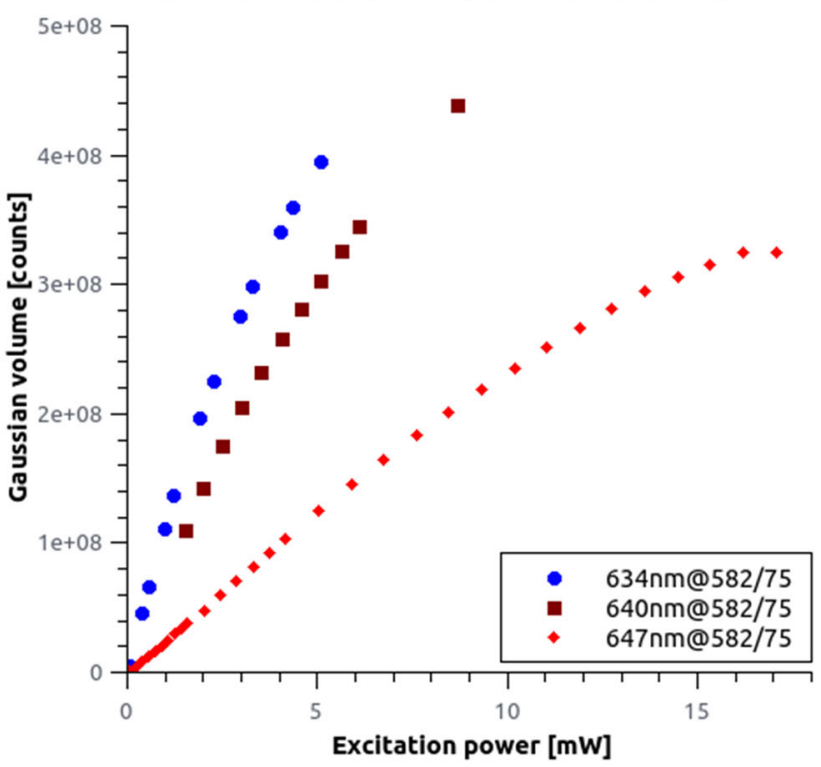

Fig. 2 Experimental measurements of the anti-Stokes fluorescence of Rh101 and AF568. The measured signal is clearly dependent on the excitation wavelength and exhibits a near-linear dependence with the excitation power. The divergence can be attributed to the saturation of the camera of the electron multiplying layer of the detector [21]. This effect can be reduced using shorter exposure times. In addition, we observed that the signal can be recorded even below a $1 \mathrm{~mW}$ excitation power, and we could not observe any threshold while decreasing the laser power. Both dyes show the same behaviour: the longer the excitation wavelength, the lower the emission rate. Based on these results, it is possible to reject the two-photon absorption model when the emission has a quadratic dependence on the excitation intensity.

\section{Fluorescence Emission Spectra}

In the second experiment, the emission spectra of Stokes and anti-Stokes fluorescence were recorded. Continuous wave laser sources (405 and $634 \mathrm{~nm}$ ) were used for excitation with two dichroic mirror and emission filter sets (Chroma ZT458rdc + Chroma ET460lp and Semrock Di01R405/488/532/635 + Semrock FF01-446/-510/581/703). Rh101 and AF568 solutions were prepared in $10^{-2} \mathrm{M}$ and $10^{-2} \mathrm{M}$ methanol solutions, respectively. The signal from the sample was coupled into a multimode fibre using a reflective collimator at the camera port and measured with an OceanOptics QE65000 spectrometer.

The results show that the normalized shape of the Stokes and anti-Stokes emission spectra highly overlap (Fig. 3). Only a slight $1-1.5 \mathrm{~nm}$ red shift is noticeable under the excitation with longer wavelengths caused by solute-solvent interaction [22-24]. For Rh101 and AF568, the maxima of the anti-Stokes emission spectra were $591 \mathrm{~nm}$ and $604 \mathrm{~nm}$, respectively. In addition, a low background was observed in the emission spectra above $700 \mathrm{~nm}$ when it was excited at $634 \mathrm{~nm}$. This was reproducible with other wavelengths and even without the fluorescent dye; thus, it was associated with the buffer. No other emission peaks were observed in the measurement range of the spectrometer. It can be assumed that the dominant relaxation path is from $S_{1}$ to $S_{0}$.

\section{Temperature-Dependent Fluorescence}

A third experiment aimed to measure the temperaturedependence of the absorption cross section. A thermal paste was applied to a glass slide, and the slide was glued to a Peltier-cooled mount (Thorlabs TCLDM9). Each dye sample was prepared in a $10^{-5} \mathrm{M}$ solution of methanol and PBS (1:1) to minimize the solvent's evaporation between the slide and cover glass. To thermally isolate the sample from the microscope frame, a dry objective (Nikon CFI S Plan Fluor ELWD 20XC) was used throughout these experiments. For excitation, a $640 \mathrm{~nm}$ wavelength pulsed laser with a repetition rate of $20 \mathrm{MHz}$ was used in a confocal line-scanning mode. The focal plane was set to approximately $1 \mu \mathrm{m}$ above the cover glass and 
(a) Rhodamine 101 normal and upconversion fluorescence

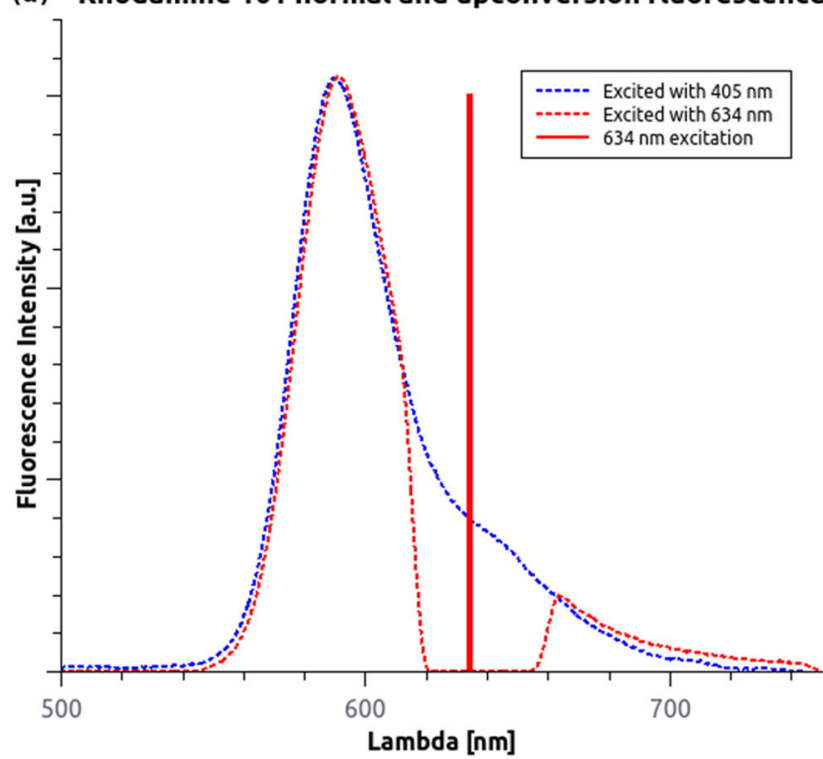

(b) Alexa Fluor $\mathbf{5 6 8}$ normal and upconversion fluorescence

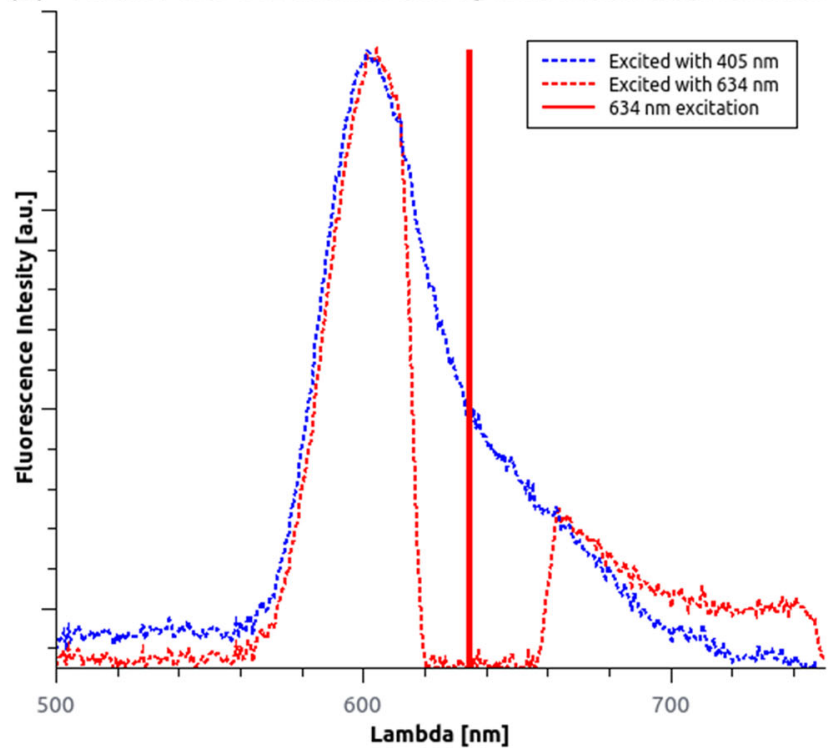

Fig. 3 Recorded Stokes- and anti-Stokes fluorescence spectra for Rh101 (a) and AF568 (b). The additional signal above $700 \mathrm{~nm}$ is related to the NIR background from the solution

was maintained by the Nikon perfect focus system. The temperature was changed gradually between $12{ }^{\circ} \mathrm{C}$ and 48 ${ }^{\circ} \mathrm{C}$ with a step size of $4{ }^{\circ} \mathrm{C}$. After the sample reached thermal equilibrium (typically $120 \mathrm{~s}$ ), a $60 \mathrm{~s}$ measurement was recorded in the two emission channels $(600 / 50$ and 690/70). The measurements were repeated several times and averaged. These multiple passthroughs over the entire temperature range in both directions eliminated the possible hysteresis effects and photobleaching. The measurements with AF568 dye were slightly affected by photobleaching after multiple heating-cooling cycles. This effect was not observable for the Rh101 dye.
A strong increase in anti-Stokes emission as a function of temperature was observed (Fig. 4). According to the theory [25], it can be assumed that the measured curves follow a Boltzmann statistic $[I(T)=A$. $\left.e^{\left(-b /\left(k_{B} \cdot T\right)\right)}\right]$, which is similar to the occupancy of the vibrational states in a single electronic band. However, we observed a linear dependence because the measurements were performed at room temperature and in a limited thermal range. The steepness of the fitted line onto the measured data was concentration-dependent because it was connected to the number of molecules in the excitation volume. The experiment was also repeated with a $560 \mathrm{~nm}$ wavelength excitation in the same temperature range used for AF568. However, the recorded intensity did not show any temperature-dependence. This observation is in good agreement with the hot-band excitation model. However, to discard the delayed fluorescence models, an additional experiment was carried out.

\section{Fluorescence Lifetime Measurements}

Fluorescence lifetimes for Stokes and anti-Stokes emissions were measured with $560 \mathrm{~nm}$ and $640 \mathrm{~nm}$ wavelength excitations. To avoid nonlinear effects and detector saturation, neutral filters with OD3 and OD1 were used in the excitation path. The repetition rate of the pulsed laser sources was set to $20 \mathrm{MHz}$. The samples from the first experiment were reused, and four FLIM measurements were recorded in the 600/50 emission channel. The acquisition was stopped when the collected number of emitted photons exceeded $10^{5}$. The arrival time distribution was fitted with a single exponential model using the Tail Fit method (SymPhoTime 64, PicoQuant).

The fitted lifetimes for the anti-Stokes fluorescence (4.193 ns; $3.56 \mathrm{~ns}$ ) were found to be consistent with those for the Stokes fluorescence (4.308 ns; $3.56 \mathrm{~ns})$ (Fig. 5). This observation discards any delayed fluorescence models. When measuring the anti-Stokes lifetime, a slight broadening of the fitted instrument response function was observed. This may indicate a very fast $(<1 \mathrm{~ns})$ relaxation path, where no intersystem crossing occurs.

\section{Potential Applications}

The spectral, lifetime and temperature dependent measurements have proved that the emitted anti-Stokes fluorescence is identical to regular Stokes shift fluorescence, therefore separation of these signals pose a challenge. In multicolour fluorescence microscopy, especially when high excitation power is applied, the reduction of spectral crosstalk is essential. AF568 is a frequently applied second dye in the dSTORM technique [26] besides AF647. Capturing image 
(a) Rhodamine 101 upconversion fluorescence $640 \mathrm{~nm}$ 20MHz OD1

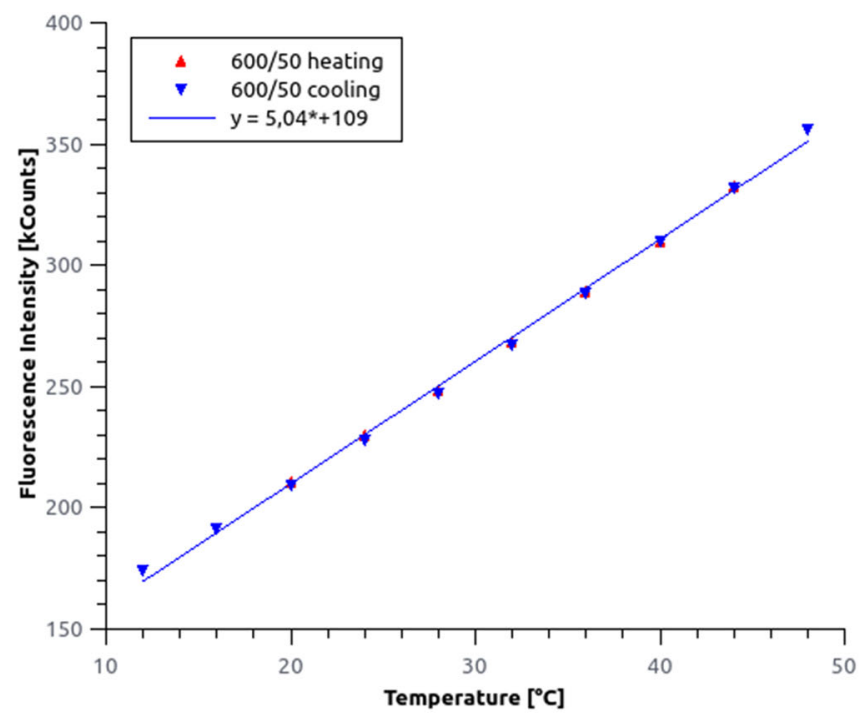

(b) Alexa Fluor 568 upconversion fluorescence $640 \mathrm{~nm}$ 20MHz OD1

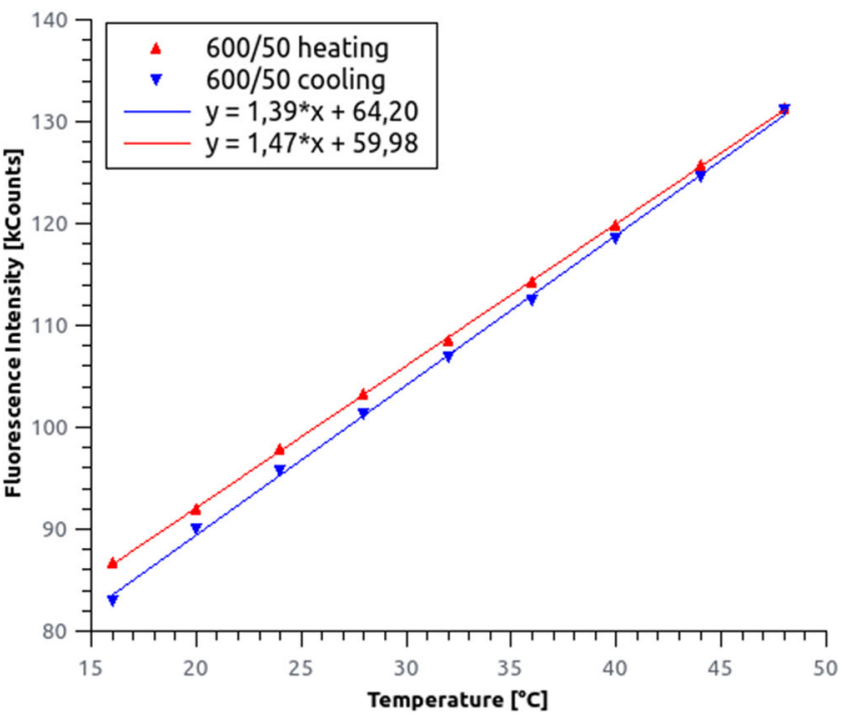

Fig. 4 Temperature-dependence of the anti-Stokes fluorescent intensity measured for R101 (a) and AF568 (b) solutions. Although a linear curve was fitted for the small measured temperature range, the hot-band excitation model predicts a Boltzmann distribution curve

(a)

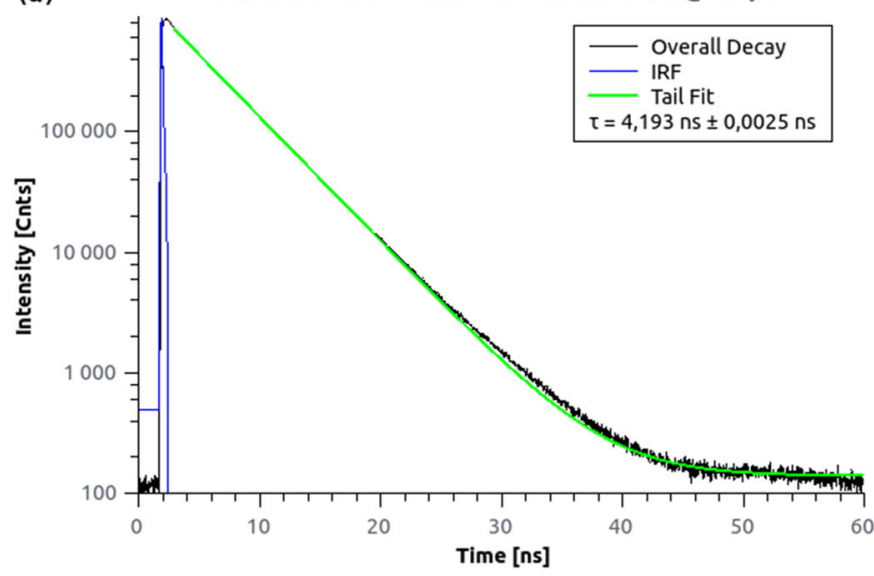

(c)

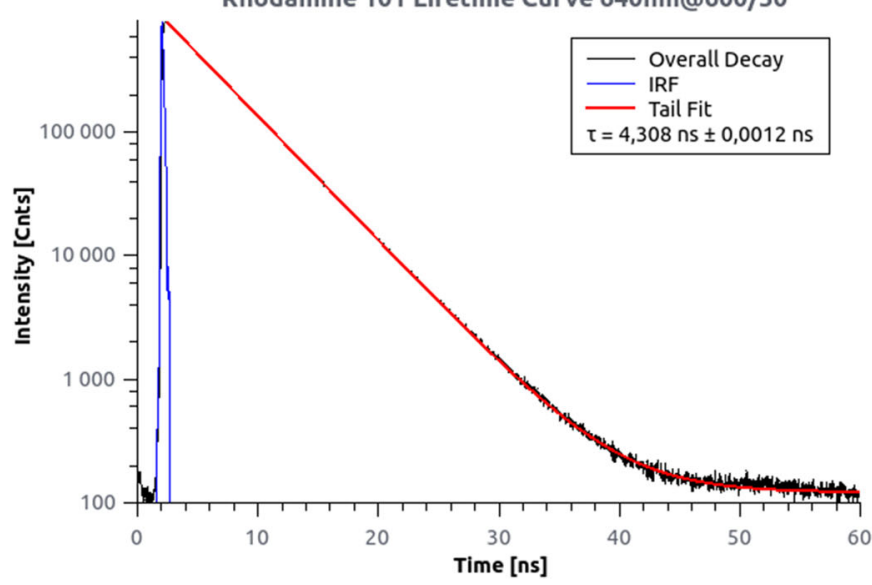

(b) Alexa Fluor 568 Lifetime Curve 560nm@600/50

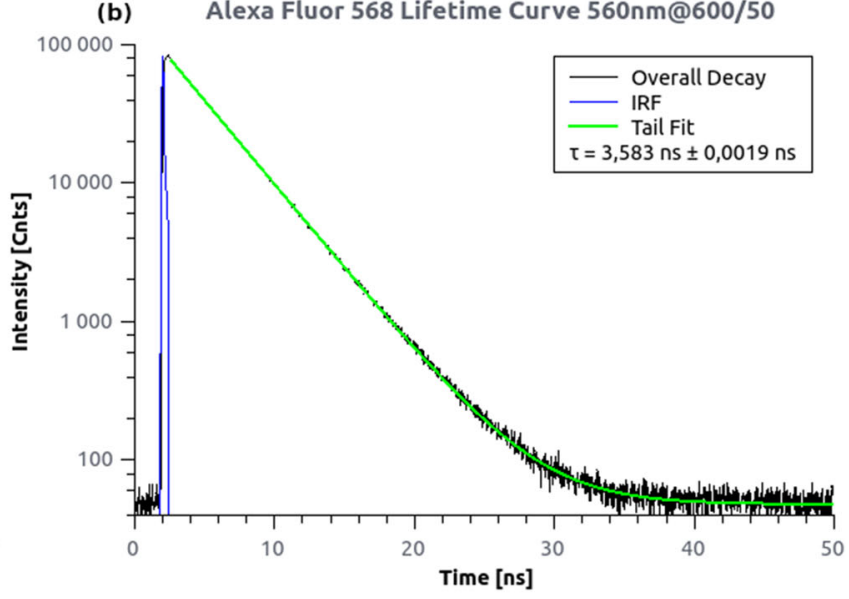

(d) Alexa Fluor 568 Lifetime Curve 640nm@600/50

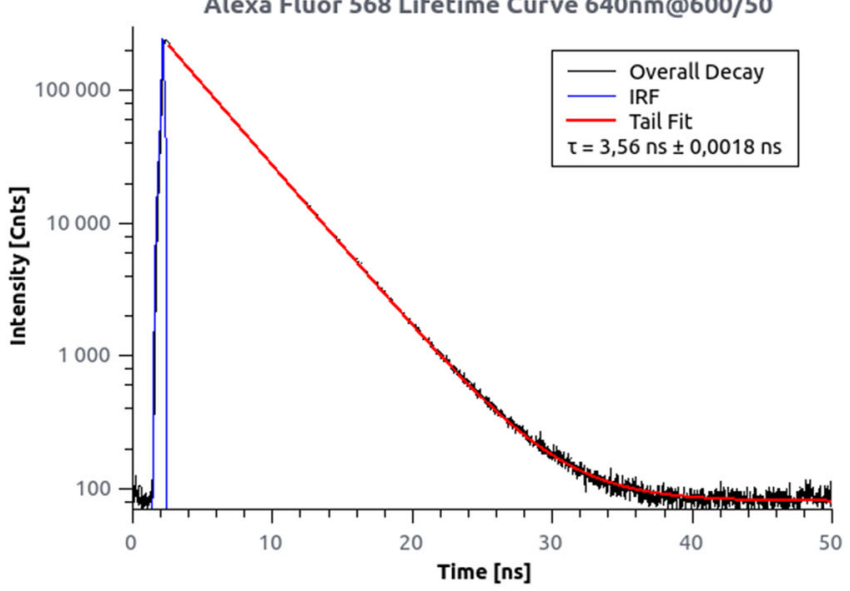

Fig. 5 Stokes and anti-Stokes fluorescent lifetime curves for Rh101 (a, c) and AF568 (b, d). A single exponential tail-fit model was used to determine the fluorescent lifetimes for each recorded arrival time 
stacks at high $\left(>3 \mathrm{~kW} / \mathrm{cm}^{2}\right)$ laser power in the far-red region can significantly increase the fluorescence background in the "green channel". This undesirable effect is typically mitigated with additional emission filters. However, the problem can be dealt with on the excitation side too, either by choosing an excitation laser with a higher wavelength (Fig. 2) or by reducing the sample's temperature (Fig. 4). In the first case the number of excitable vibrational states, while in the second one the occupancy of the vibrational states is lowered. However, anti-Stokes fluorescence can be used in multiple ways when captured in a separate spectral window (600/50) simultaneously with the main emission channel (690/70) (e.g. Cairn OptoSplit II, or Hamamatsu W-View Gemini). Here we discuss three potential application areas of the anti-Stokes fluorescence of AF568.

During dSTORM super-resolution measurements the lateral and axial drift can reduce the final image quality. The axial drift is typically minimized by using focus keeping hardware, and the lateral one can only be removed with software-based solutions (using fiducial markers or by correlating the localizations in time). However, the density and spatial distribution of fiducial markers on the sample cannot be controlled. They also introduce a high intensity light source, precluding the localization of adjacent single dye emitters. Fluorescent beads labelled with hotband active dyes (e.g. Rh101 or AF568) can be used to address this issue. The lateral drift can be corrected by simultaneously recording the anti-Stokes signal in a separate spectral channel. A similar approach using only one emission window has been already proposed [27]. In addition, the approach can be further developed to correct the axial drift in astigmatic 3D localization microscopy, when the elliptical shape of the PSF provides information on the axial position.

The reduced FOV $\left(<20 \times 20 \mu \mathrm{m}^{2}\right)$ and finite lifetime of the switching buffer ( $<4$ hours) in localization based microscopy methods make the selection of ROI a difficult task. Labelling the interesting cells, cell components, structures or proteins in the sample with a different dye can be used to significantly reduce searching time. Hot-band active dyes are an ideal choice for such applications since the two spectral channels can be captured simultaneously using the same laser source. For example, the axon initial segment of a neuron in a brain tissue experiment can be found by labelling the $\beta I V$ spectrin [28]. In a muscle cell culture desmin accumulation is a key indicator for differentiation [29, 30], while in myofibrils the elastic protein components (kettin, Sls700 etc.) related to the Iband can be marked [20].

Autofluorescence is a critical limitation in fluorescence microscopy. It reduces the signal-to-noise ratio and can introduce artefacts. Several sample preparation protocols and histochemical techniques [31] have been proposed to eliminate or reduce such undesirable effects. Since autofluorescence is typically more significant at the low wavelength range (due to the high photon energy), the application of far-red dyes can be a possible solution too. However, hot-band active dyes can also be used for reducing autofluorescence. Samples labelled with AF568 and excited by red lasers (e.g. $647 \mathrm{~nm}$ ) can be imaged with reduced autofluorescence signal. In this case only the hot-band emission signal of the fluorescent dye can be captured [32], and the autofluorescence background in the far-red region can be spectrally separated (see Fig. 3). A similar signal-tonoise ratio enhancement has been already demonstrated via upconverting nanoparticles [33, 34].

\section{Conclusion}

Comparative time-resolved and steady-state measurements of the anti-Stokes fluorescence emission of AF568 have revealed that the emission intensity linearly depends on both the excitation power and temperature in the studied ranges. No intensity threshold was detected and both the emission spectrum and the fluorescence lifetime were found to be identical to the normal Stokes fluorescence values. These findings have confirmed that the detected anti-Stokes fluorescence of AF568 is the consequence of the hot-band absorption-emission pathway. The undesirable anti-Stokes emission rate can be reduced by manipulating either the temperature or the excitation wavelength. However, several potential applications have been suggested where hot-band active dyes can be used for drift correction, fast ROI selection or autofluorescence reduction.

Acknowledgements This work was supported by the Hungarian Brain Research Program (2017-1.2.1-NKP-2017-00002); the GINOP-2.3.215-2016-00036; the EU-funded Hungarian Grant EFOP-3.6.1-162016-00008. We thank Gábor Laczkó for a fruitful discussion. We would like to acknowledge József Mihály and Szilárd Szikora for providing the drosophila myofibril sample. Open access funding provided by University of Szeged (SZTE).

\section{Compliance with Ethical Standards}

Conflict of interests The authors have declared that no conflicting interests exist.

Open Access This article is licensed under a Creative Commons Attribution 4.0 International License, which permits use, sharing, adaptation, distribution and reproduction in any medium or format, as long as you give appropriate credit to the original author(s) and the source, provide a link to the Creative Commons licence, and indicate if changes were made. The images or other third party material in this article are included in the article's Creative Commons licence, unless indicated otherwise in a credit line to the material. If material is not included in the article's Creative Commons licence and your intended use is not permitted by statutory regulation or exceeds 
the permitted use, you will need to obtain permission directly from the copyright holder. To view a copy of this licence, visit http:// creativecommonshorg/licenses/by/4.0/.

\section{References}

1. Kasha M (1950) Discuss Faraday Soc 9:14. https://doi.org/10. 1039/DF9500900014

2. Stokes GG (1852) Philos Trans R Soc London 142:463. https://doi.org/10.1098/rstl.1852.0022

3. Landau L (1946) J Phys (Moscow), 10(503). https://doi.org/10. 1016/B978-0-08-010586-4.50067-5

4. Göppert-Mayer M (1931) Ann Phys 401(3):273. https://doi.org/ 10.1002/andp.19314010303

5. Kaiser W, Garrett C (1961) Phys Rev Lett 7(6):229. https://doi. org/10.1103/PhysRevLett.7.229

6. Parker C, Hatchard C (1963) Trans Faraday Soc 59:284. https://doi.org/10.1039/TF9635900284

7. Birks J (1963) J Phys Chem 67(10):2199. https://doi.org/10. $1021 / \mathrm{j} 100804 \mathrm{a} 054$

8. Parker C, Hatchard C (1961) Trans Faraday Soc 57:1894. https://doi.org/10.1039/TF9615701894

9. Clark J, Miller P, Rumbles G (1998) J Phys Chem A 102(24):4428. https://doi.org/10.1021/jp980589c

10. Erickson L (1972) J Lumin 5(1):1. https://doi.org/10.1016/00222313(72)90030-0

11. Kachynski A, Kuzmin A, Pudavar H, Prasad P (2005) Appl Phys Lett 87(2):023901. https://doi.org/10.1063/1.1993761

12. Jain R, Hu C, Gustafson T, Elliot S, Chang M (1973) J Appl Phys 44(7):3157. https://doi.org/10.1063/1.1662724

13. Kumazaki S (2013) Chem Phys 419:107. https://doi.org/10.1016/ j.chemphys.2013.02.030

14. Meshalkin YP, Svetlichnyi V, Lapin I (2007) Russ Phys J 50(3):267. https://doi.org/10.1007/s11182-007-0037-0

15. Epstein R, Buchwald M, Edwards B, Gosnell T, Mungan C (1995) Nature 377(6549):500. https://doi.org/10.1038/377500a0

16. Clark J, Rumbles G (1996) Phys Rev Lett 76(12):2037. https://doi.org/10.1103/PhysRevLett.76.2037

17. Mungan C, Buchwald M, Edwards B, Epstein R, Gosnell T (1997) Phys Rev Lett 78(6):1030. https://doi.org/10.1103/ PhysRevLett.78.1030

18. Luo X, Eisaman M, Gosnell T (1998) Opt Lett 23(8):639. https://doi.org/10.1364/OL.23.000639
19. Novák T, Gajdos T, Sinkó J, Szabó G, Erdélyi M (2017) Sci Rep 7(1):951. https://doi.org/10.1038/s41598-017-01122-7

20. Szikora S, Gajdos T, Novák T, Farkas D, Földi I, Lenart P, Erdélyi M, Mihály J (2020) J Cell Biol, 219(1). https://doi.org/10.1083/jcb.201907026

21. Wang X, Kress A, Brasselet S, Ferrand P (2013) Rev Sci Instrum 84(5):053708. https://doi.org/10.1063/1.4807318

22. Azumi T, Itoh Ki, Shiraishi H (1976) J Chem Phys 65(7):2550. https://doi.org/10.1063/1.433440

23. Demchenko AP (2002) Luminescence: J Biol Chem Luminescence 17(1):19. https://doi.org/10.1002/bio.671

24. Tomin VI (2010). In: Advanced fluorescence reporters in chemistry and biology I. Springer, pp 189-223, https://doi.org/10. 1007/978-3-642-04702-2_6

25. Guardigli M (2012) Anal Bioanal Chem 403(1):1. https://doi.org/ 10.1007/s00216-012-5787-z

26. Erdelyi M, Rees E, Metcalf D, Schierle GSK, Dudas L, Sinko J, Knight AE, Kaminski CF (2013) Opt Express 21(9):10978. https://doi.org/10.1364/OE.21.010978

27. Balinovic A, Albrecht D, Endesfelder U (2019) J Phys D: Appl Phys 52(20):204002. https://doi.org/10.1088/1361-6463/ab0862

28. Huang CYM, Zhang C, Ho TSY, Oses-Prieto J, Burlingame AL, Lalonde J, Noebels JL, Leterrier C, Rasband MN (2017) J Neurosci 37(47):11311. https://doi.org/10.1523/JNEUROSCI.211217.2017

29. Li H, Capetanaki Y (1993) Nucleic Acids Res 21(2):335. https://doi.org/10.1093/nar/21.2.335

30. Gunetti M, Tomasi S, Giammò A, Boido M, Rustichelli D, Mareschi K, Errichiello E, Parola M, Ferrero I, Fagioli F et al (2012) PloS one 7(9):e45538. https://doi.org/10.1371/journal. pone.0045538

31. Baschong W, Suetterlin R, Laeng RH (2001) J Histochem Cytochem 49(12):1565. https://doi.org/10.1177/0022155401049 01210

32. Zhu X, Su Q, Feng W, Li F (2017) Chem Soc Rev 46(4):1025. https://doi.org/10.1039/c6cs00415f

33. Chatterjee DK, Rufaihah AJ, Zhang Y (2008) Biomaterials 29(7):937. https://doi.org/10.1016/j.biomaterials.2007.10.051

34. Zhou JC, Yang ZL, Dong W, Tang RJ, Sun LD, Yan CH (2011) Biomaterials 32(34):9059. https://doi.org/10.1016/j.biomaterials. 2011.08.038

Publisher's Note Springer Nature remains neutral with regard to jurisdictional claims in published maps and institutional affiliations. 